\title{
Porous Shape-Memory NiTi-Nb with Microchannel Arrays
}

\author{
C. Bewerse ${ }^{1}$, L.C. Brinson ${ }^{1,2}$, D.C. Dunand ${ }^{1 *}$
}

${ }^{1}$ Department of Materials Science and Engineering, Northwestern University, 2220 Campus Drive, 2036, Evanston, IL 60208.

${ }^{2}$ Department of Mechanical Engineering, Northwestern University, 2145 Sheridan Road, Room B224, Evanston, IL 60208.

* Corresponding author. dunand@northwestern.edu. Ph: 847/491-5370

\begin{abstract}
Porous NiTi-Nb containing a 3D array of orthogonally interconnected microchannels was created via a novel powder metallurgy process combining: (i) Mg ribbon scaffold construction, (ii) slip casting of $\mathrm{NiTi+Nb}$ powder blend within the scaffold, (iii) $\mathrm{Mg}$ scaffold vacuum evaporation, and (iv) NiTi liquid phase sintering. The later stage was achieved by creating small amounts of quasi-binary NiTi-Nb liquid eutectic, which wicked between NiTi particles and bonded them together while leaving 28 vol.\% of residual pores. These hierarchical porous structures have a total porosity of 30-53\%, an effective stiffness of 5-9 GPa, and a yield strength of 20-80 MPa. They exhibit the shape memory effect, with 3\% strain recovery after $7 \%$ compressive deformation upon multiple load-unload cycles. FEM is used to model the anisotropy of these structures, as well as to probe the strain distributions on a microscopic level. Mechanical anisotropy was also present in FEM of all structures, though more pronounced in structures with high microchannel volume fraction. With mechanical properties between those of trabecular and cortical bone, these structures are of great interest for bone implant applications.
\end{abstract}

\section{Introduction}


NiTi alloys near the equiatomic composition are desirable for their shape memory deformation behavior, where 5-7\% strain can be recovered through a diffusionless, reversible phase transformation [1-3]. NiTi alloys have good strength and toughness, and are biocompatible [4], making them attractive for biomedical implant devices [5], actuators [6], and energy damping structures [7]. NiTi porous structures are particularly attractive for bone implant applications, as the porosity lowers the structural stiffness closer to that of cortical or trabecular bone and allows bone ingrowth into the structure for osseointegration [8]. Powder processing methods to make NiTi foams have been reviewed by Ryan et al. [9] as well as Bansiddhi et al. [4], specifically for the fabrication of NiTi implants by Elahinia et al. [2], and for total joint replacement applications by Nasab et al. [10].

Porous NiTi structures have been created by combining the space holder technique with various powder metallurgy methods to bond NiTi powders. Salt spaceholders powders such as $\mathrm{NaCl}$ [11] and $\mathrm{NaF}$ [12], later removed by dissolution in water, were used with hot isostatic pressing to create NiTi structures with $20-40 \%$ porosity, and stiffnesses between 4 and $10 \mathrm{GPa}$. Hot isostatic pressing of NiTi powders containing steel mesh spaceholders was also utilized to create NiTi structures with 2D interconnected wavy microchannels [13] with porosities ranging between 24 and $34 \%$ and anisotropic mechanical properties with compressive stiffnesses between 15 and $35 \mathrm{GPa}$, and strengths between 430 and $780 \mathrm{MPa}$. Conventional sintering of NiTi/Mg powders blends also produced structures with $40-80 \%$ porosity [14]. The Mg powder spaceholder was removed by evaporation, and the $\mathrm{Mg}$ vapor atmosphere was found to enhance the sintering of the NiTi powders [15]. These structures exhibited excellent mechanical properties for biomedical applications, with a low stiffness of 6-13 GPa and a yield stress of 90$155 \mathrm{MPa}$ [16]. 
Liquid phase sintering has been used to improve bonding of numerous types of metallic powder without pressure or combustion [17]. At a nominal composition of Ti- $40 \mathrm{Ni}-20 \mathrm{Nb}$ at $\%$, there is a eutectic point at $1150^{\circ} \mathrm{C}$ [18-20]. Thus, by adding small amounts of $\mathrm{Nb}$ to prealloyed NiTi powders, a small volume of liquid eutectic forms by reaction between the $\mathrm{Nb}$ and some of the NiTi powders when the powder blend is heated above the eutectic temperature [21]. This liquid eutectic wicks between the remaining, unreacted NiTi powders, bonding them together upon solidification. Liquid phase sintering has also been combined with spaceholders such as $\mathrm{NaCl}$ [11] or $\mathrm{Nb}$ wire spaceholders, [22], creating structures with porosities between 40 and 60\%. This technique has also been used for brazing of NiTi strips to create honeycombs [23-25] and other materials such as $\mathrm{Nb}$-containing TiAl alloys [26] and $\mathrm{C} / \mathrm{Si}$ composites [27].

Slip casting [28] has been used to create Ti and NiTi foams with sodium alginate as a binder, by partial sintering of Ti powders [29] and by SHS of elemental Ni and Ti powders [30], respectively. In another study, the slurry to make Ti foams was optimized using a PVA binder [31]. A NiTi slurry technique with a PVA binder was also developed by immersing a polymer sponge in the slurry, followed by conventional sintering to remove the polymer sponge and form a cellular foam [32]. These structures had a porosity of $65-72 \%$ with a stiffness of $3 \mathrm{GPa}$, but failed at a low total strain of $3.5 \%$ at $73 \mathrm{MPa}$.

Here, we combine several of the techniques reviewed above into a novel method to create NiTi$\mathrm{Nb}$ porous structures with $3 \mathrm{D}$ interconnected microchannels. A NiTi-Nb blend slurry with a PVA binder is cast around and within a scaffold of Mg ribbons to form a green composite. PVA is chosen as a binder for its low thermal decomposition temperature [31]. Magnesium is also used as a spaceholder for its ease of removal via evaporation $[14,15]$. In a multi-step heat treatment under vacuum, the PVA binder and Mg spaceholders are removed, and the NiTi-Nb 
powder preform is bonded through liquid phase sintering. The slip casting process is pressureless and fully scalable as compared to processing technique that require specialized, costly equipment such as hot-isostatic pressing or MIM. The Mg ribbon spaceholder is also commercially available, inexpensive and easily removable without contamination of NiTi, while the low melting temperature of the NiTi-Nb eutectic allows for a simplified processing. In addition, the established biocompatibility of $\mathrm{Nb}$ [33] and NiTi [4], as well as good corrosion resistance for $\mathrm{NiTi-Nb}$ [34], is desirable for using these structures in biomedical implants.

\section{Experimental Methods}

Prealloyed NiTi powders were used to prevent formation of undesirable intermetallic phases, as in previous studies using elemental $\mathrm{Ni}$ and Ti powders [12,31]. The NiTi powders were gas atomized (Special Metals Corp. Inc) with a nominal composition of 51.4 at $\%$ Ti and sieved to a range of $44-63 \mu \mathrm{m}$. NiTi powders were blended with $5.3 \mathrm{wt} . \% \mathrm{Nb}$ powders $(1-5 \mu \mathrm{m}$, Alfa Aesar) by tumbling for $2 \mathrm{hr}$ in a $40 \mathrm{~mL}$ glass bottle. Magnesium ribbon (Alfa Aesar) with a cross-section of $3 \times 0.3 \mathrm{~mm}$ was chosen as a spaceholder. The ribbon was cut into $19 \mathrm{~mm}$ long strips which were stacked in a plaster mold. Molds (Fig. 1A) were cast from plaster, with a plaster to water ratio of 2:1, with grooves for alignment of the magnesium ribbon. The grooves were $0.5 \mathrm{~mm}$ wide and spaced at $0.5 \mathrm{~mm}$ intervals.

Specimens with three levels of porosity were created, designated in the following as monolithic (M), low porosity (LP), and high porosity (HP). The monolithic samples contained no magnesium spaceholder. LP samples contained 15 vol.\% of magnesium in the green structure, with ribbons placed in every other insert groove for a nominal center-to-center spacing of $2 \pm 0.2$ $\mathrm{mm}$. For HP samples, magnesium ribbons were placed in every groove for a nominal $1 \pm 0.2$ $\mathrm{mm}$ center-to-center spacing, resulting in 30 vol.\% magnesium spaceholder in the green 
structure. Magnesium ribbons were placed in four layers within the plaster inserts with orthogonal stacking. In the LP samples, there were seven magnesium ribbons in each layer, and twice this number in each HP layer.

Polyvinyl alcohol (PVA) - with an average molecular weight of 13,000-23,000, from Sigma Aldrich - was chosen as binder because of its low decomposition temperature, so that oxidation of $\mathrm{NiTi}$ or $\mathrm{Nb}$ powders could be minimized during gas evolution. The slurry for slip casting was composed of 75 wt.\% (dry) blended NiTi-Nb powders with 0.8 wt.\% (dry) PVA and a balance of deionized water. This binder concentration was chosen based on Ref. [31], where 0.8 wt.\% (dry) PVA binder was found to be sufficient for handling of the green samples. The PVA was first dissolved in deionized water at $140{ }^{\circ} \mathrm{C}$, and then the NiTi-Nb powder blend was stirred into the solution. The slurry was then poured into the plaster mold stacked with magnesium ribbons for $\mathrm{LP}$ and HP samples, or without $\mathrm{Mg}$ in the $\mathrm{M}$ sample. The green samples were dried at $130^{\circ} \mathrm{C}$ overnight and then removed from the plaster mold. An example of a green HP sample is shown in Fig. 1B.

The green samples were then sintered in a three-step heat treatment performed in a high vacuum tube furnace under a residual pressure of $10^{-6}$ torr. The PVA binder was first decomposed at $330^{\circ} \mathrm{C}$ for $2 \mathrm{~h}$. The magnesium spaceholder was then evaporated while ramping to $1100{ }^{\circ} \mathrm{C}$ at a rate of $10^{\circ} \mathrm{C} /$ minute, followed by a partial solid-state sintering of the NiTi-Nb powders at $1100^{\circ} \mathrm{C}$ for $1 \mathrm{~h}$. The structure was then liquid-phase sintered at $1180^{\circ} \mathrm{C}$ (above the $\mathrm{NiTi}-\mathrm{Nb}$ eutectic temperature of $1148{ }^{\circ} \mathrm{C}$ [26]) for 30 minutes. The samples were finally furnace-cooled at a rate of $7^{\circ} \mathrm{C} /$ minute.

To characterize mechanical anisotropy, ten parallelepipeds with dimensions of $5 \times 5 \times 10 \mathrm{~mm}$ were cut by electric discharge machining (EDM) from the solidified samples with two different 
specimen orientations. Samples were cut such that the angle between the long direction of the parallelepiped and the stacking direction of the evaporated magnesium spaceholder was $0^{\circ}$ or $90^{\circ}$ for both LP and HP samples. This resulted in four geometries (in addition to the M specimens): $0^{\circ}$ and $90^{\circ}$ orientations for LP and HP samples which are schematically displayed in Fig. 1C-F. A schematic of the full HP sample is illustrated in Fig. 1G, with the HP0 and HP90 specimens outlined. These specimens are referred to by their porosity and orientation throughout the paper. For example, a LP sample with $0^{\circ}$ angle between the long direction of the specimen and the spaceholder stacking direction will be referred to as LP0. Prior to mechanical testing, the faces of the specimen were lightly ground with 600 grit grinding paper to remove the thin oxide layer formed during the EDM process.

Compression testing was performed on a screw-driven load frame (Sintech 20/G) using an alignment cage with tungsten carbide inserts to ensure that the sample contact surfaces were parallel during all loading and unloading. Strain was calculated from crosshead displacement with compliance correction subtracted using the direct method [35]. The specimens were tested at a rate of $1 \times 10^{-4} \mathrm{~s}^{-1}$ under either monotonic loading to failure or cyclic loading-unloading conditions. During cyclic compression, the specimen was loaded to a predetermined stress, unloaded, and temperature-cycled to achieve SMA recovery, before being subjected to the next load-unload cycle to a higher predetermined stress. The incremental maximum loads for each cycle were determined from the monotonic loading curve, corresponding to $1 \%$ strain increments. The temperature-cycle, also performed on samples prior to any mechanical testing, was as follows: 10 minutes at $130^{\circ} \mathrm{C}$ in an air box furnace, followed by cooling to $-196^{\circ} \mathrm{C}$ in a liquid nitrogen bath. The samples were then allowed to return to room temperature before mechanical testing. 
Metallographic preparation of the monolithic slip-cast matrix was performed on a cross-sections cut with a low-speed diamond saw by mounting, grinding with 400, 800, and 1200 grit grinding paper, and polishing with $0.05 \mu \mathrm{m}$ colloidal silica suspension. The cross-section was cut parallel to the stacking direction. Scanning Electron Microscopy (Hitachi S-3400) with Energy Dispersive X-Ray Spectroscopy (EDX) was used to evaluate the microstructure and phases of the polished sample, as well as the macroscopic structure of the machined samples prior to, and after, compression. The total porosity of each specimen was measured through the Archimedes method using $6.56 \mathrm{~g} / \mathrm{cm}^{3}$ as the density of the liquid-sintered NiTi-Nb (calculated using the rule of mixtures with the density of bulk NiTi as $6.45 \mathrm{~g} / \mathrm{cm}^{3}$ [36] and the density of $\mathrm{Nb}$ as $8.57 \mathrm{~g} / \mathrm{cm}^{3}$ ). The open pores were vacuum-infiltrated with oil and then sealed with grease prior to measurement of the porosity. Closed porosity within the liquid sintered matrix was calculated by measuring the volume of the matrix with helium pycnometry (AccuPyc 1300). The matrix microporosity and the microchannels in LP and HP samples were also examined optically on polished cross-sections, with micrographs converted to binary images where pores are black and the metallic matrix is white.

Transformation temperatures were measured by differential scanning calorimetry by ATI Wah Chang Analytical Laboratory. Samples were heated and cooled though a range of $-100^{\circ} \mathrm{C}$ to $125^{\circ} \mathrm{C}$ at a rate of $10^{\circ} \mathrm{C} /$ minute under nitrogen gas cover. The austenite and martensite start and finish temperatures ( $A_{S}, M_{S}, M_{F}$, and $M_{F}$, respectively) were determined as the intersection of the tangent points of the peak and the baseline of the temperature vs. heat flow curves, while the transformation enthalpies were determined as the area under the peak. Carbon and oxygen content in the samples were also measured by ATI Wah Chang Analytical Laboratory.

\section{Results and Discussion}




\subsection{Microstructure}

The monolithic slip cast structure has a porous microstructure with $27.6 \pm 0.5 \%$ total porosity, of which $26.9 \pm 0.5 \%$ is open and $0.7 \pm 0.2 \%$ is closed porosity. NiTi powder packing is not uniform during the slip casting process, and the powders are not densified by compaction, as in other dry-powder processes. The only densification of the powders is due to water tension as the water wicks from the slip cast green structure into the plaster mold. If the NiTi powders had been fully densified, the PVA binder would account, by mass conversion, for only 5.4 vol.\% of the green samples. Thus, the additional porosity in the slip cast matrix is due to the space between the NiTi/Nb powder particles. During the liquid phase sintering process, the eutectic liquid wicks between the remaining NiTi powders and the space present initially between the powder particles partially coalesces into larger voids, such as that outlined with dotted line in Fig. 2B, or remains as irregular smaller pores as marked with arrows in Fig. 2B. This process results in a majority of open porosity and only a minor amount of closed porosity. Closed porosity only forms when the solidified eutectic completely encloses a region of porosity between NiTi powders. The microporosity of the matrix could be reduced by increasing the solid-sintering step time, by increasing the amount of eutectic liquid, or by using a $\mathrm{NiTi}-\mathrm{Nb}$ powder blend with a bimodal range of diameters to achieve a higher packing factor. Cold isostatic pressing of the green preform is also a possible solution.

Fig. $2 \mathrm{C}$ shows a magnified view of one of the eutectic bridges between two NiTi powders. The NiTi powders are outlined with a dashed line, though it is not a discrete interface. The Nb-rich lamellae structure of the eutectic is visible as a lighter phase contrast on the backscattered SEM micrograph due to the difference in atomic mass between the elements, with phases rich in $\mathrm{Nb}$, which has a higher atomic mass than $\mathrm{Ni}$ or $\mathrm{Ti}$, showing lighter contrast [21]: this eutectic 
lamellar phase has been determined to be bcc-Nb, with $5-19$ at.\% $\mathrm{Ti}$ and $5-10$ at.\% $\mathrm{Ni}$ $[19,20,24]$. Two of these lamellae are outlined in Fig. 2D within the eutectic bridge, but the lamellae are not limited to the eutectic bridge. $\mathrm{Nb}$ is also found on the surface of the NiTi powders away from the bridge, such as one marked with an arrow in Fig. 2C. This indicates that the eutectic wets the entire surface of the NiTi powders, rather than just the connection between powders.

\subsection{Macrostructure}

After liquid phase sintering, the $\mathrm{Mg}$ ribbon spaceholders were completely removed and replicated as continuous microchannels. The microchannels extended fully through the structures, as observed by visual inspection against a lighted background. Interconnection windows were formed between orthogonal layers of microchannels at the contact points of the orthogonally stacked Mg ribbons. Fig. 2A is a SEM micrograph of the exposed inner surface of a microchannel sectioned by EDM, showing the interconnection windows between one exposed microchannel (horizontal in the micrograph) and six orthogonal microchannels (vertical in the micrograph) in the adjacent layer. Arrows mark in Fig. 2A four of the intersections between orthogonal microchannels which have well defined windows; one microchannel intersection (second from right, circled) is closed and another one (second from left) is partially closed. During processing, the $\mathrm{Mg}$ ribbons were loosely stacked in the grooves within the plaster mold, with no physical attachment between them. The closed microchannel intersection may have been caused by incomplete contact between $\mathrm{Mg}$ ribbons during stacking, resulting in $\mathrm{NiTi}-\mathrm{Nb}$ PVA slurry filling in the intersection point and solidifying during liquid phase sintering into a closed intersection. 
The microchannel surface is macroscopically flat, as illustrated in Fig. 2A, though textured as compared to the machined surface. During liquid sintering, the liquid eutectic wicked into the matrix structure without filling the microchannels previously created by evaporation of the $\mathrm{Mg}$ ribbons. The residual NiTi powders previously in contact with the Mg ribbons remain as the surface of the microchannel, creating the texture visible in Fig. 2A.

The microchannel width was measured on SEM micrographs to be $255 \pm 30 \mu \mathrm{m}$, reduced from the $300 \mu \mathrm{m} \mathrm{Mg} \mathrm{ribbon} \mathrm{thickness.} \mathrm{Microchannel} \mathrm{dimensions} \mathrm{were} \mathrm{measured} \mathrm{from} \mathrm{machined}$ specimen faces. Though the cuts are nominally parallel or perpendicular to the microchannels, any deviation from orthogonality will cause variation in the observed width of the rectangular microchannel cross-section. During liquid phase sintering, formation of the liquid could also cause slumping of the channel walls, decreasing the microchannel width from the Mg ribbon thickness, or may induce uniform shrinkage of the sample. It is likely that each effect contributes to the reduction in microchannel width. For LP samples, the thickness of the walls separating the microchannels is $1340 \pm 70 \mu \mathrm{m}$, while for HP samples the wall thickness is $520 \pm$ $120 \mu \mathrm{m}$, as measured from the machined specimen faces. This is smaller than the Mg ribbon spacing in the green structure before the heat treatment, which was $1700 \mu \mathrm{m}$ for LP samples and $700 \mu \mathrm{m}$ for HP samples, indicating shrinkage from powder densification.

Each of the HP and LP structures had microchannel porosity in addition to the matrix porosity (28\% porous matrix). The total porosities of the structures, as measured with Archimedes testing, are listed in Table 1. Considering a microchannel width of $255 \mu \mathrm{m}$, microchannel walls of $520 \mu \mathrm{m}$ and $1340 \mu \mathrm{m}$ for HP and LP structures respectively, and a matrix with $28 \%$ porosity, the theoretical porosity of a bulk HP or LP structure can be calculated. For the theoretical LP structure, the structure porosity is $41 \%$, of which $16 \%$ is microchannel porosity. For the 
theoretical HP structure, the total structure porosity is $53 \%$, of which $33 \%$ is microchannel porosity. The actual matrix and microchannel porosities are listed in Table 1. These vary from the theoretical structure porosities, though similar, due to machining limitations resulting in nonideal structures.

\subsection{Mechanical properties}

\subsubsection{Monotonic Loading of Slip Cast Structures}

The stress strain curves for monotonically loaded specimen are shown in Fig. 3A. The slip cast $\mathrm{NiTi-Nb}$ monolithic foam specimen $\mathrm{M}$ exhibited low stiffness of $9.0 \pm 0.6 \mathrm{GPa}$ with SMA properties. SMA martensite orientation initiated at $1.0 \%$ strain at a stress of $80 \mathrm{MPa}$, as measured by the interception of the stiffness slope and the plateau slope. While bulk NiTi typically has a relatively horizontal shape memory plateau, the plateau here is graded with a slope. This feature is similar to that found in literature of other NiTi-Nb liquid sintered materials, both pseudo bulk (6\% porous) and foams (34-44\% porosity) [21]. The stiffness and strength of specimen $\mathrm{M}$ is between that of trabecular and cortical bone. Cortical bone has a higher stiffness, between 12 and $25 \mathrm{GPa}$, while trabecular bone is a full order magnitude lower in stiffness, between 0.01 and $0.9 \mathrm{GPa}$ [37]. The compressive strength is also lower than for cortical bone $(133$ - $193 \mathrm{MPa})$, but again an order of magnitude higher than for trabecular bone $(0.6-9.3 \mathrm{MPa})[37]$. This indicates that the slip cast NiTi-Nb structure would be appropriate for transition regions between cortical and trabecular bone, or for trabecular bone regions. Depending on the location of the implant, as mechanical properties are still similar to cortical bone, the structure here might still be appropriate for bone replacement.

Hardening was apparent near $4.0 \%$ strain, and densification continued to $28.3 \%$ strain at $900 \mathrm{MPa}$ at the limit of the load cell though collapse of the cellular structure, with no spalling or critical 
failure on the macroscopic level. After unloading, the specimen was heated through the phase transformation and recovered $0.8 \%$ strain through the Shape memory effect (SME), with $24.4 \%$ residual plastic strain. This strain recovery is low as compared to bulk NiTi in compression, which recovers 3-5\% strain [38]. SMA recovery is reduced within the slip cast porous structure, as the microscopic strain recovery within the NiTi powders is constrained by local plasticity. With lower amounts of plasticity, as examined with load-unload-recovery cycles later in Fig. 3B, $3 \%$ strain is recovered through the SME. However with increasing plasticity, the reduction in SMA recovery becomes more pronounced, as seen in the present study. At high levels of strain, the macroscopic strain recovery is also reduced by cracking and failure of the eutectic bridges connecting the NiTi powders, preventing local strain recovery from translating to macroscopic strain recovery.

The monotonic stress-strain curves for the hierarchical structures with microchannels - LP90, LP0, HP90, and HP0 - are plotted in Fig. 3A. The full stress-strain curve for HP90 is shown as an inset. HP90 was loaded to higher strains because it did not exhibit critical failure through a sudden load drop, as the other structures did. All of the structures have low stiffness, ranging from $4.3 \pm 0.2 \mathrm{GPa}$ (HP90) to $6.8 \pm 0.1 \mathrm{GPa}$ (LP90), which are listed in Table 1. The structures also all have a lower yield stress as compared to M, ranging from $20-65 \mathrm{MPa}$. Both the LP and HP structures exhibit anisotropy in the stress-strain curve between the 0 and 90 samples. The shape of the two LP structures are both similar to M, exhibiting a SMA plateau, while the deformation of the HP structures is more affected by the geometry of the channels. The LP structures both densify without buckling or spalling of the matrix, similar to M. However the LP90 structure, with the microchannel stacking direction perpendicular to the loading direction, exhibits a higher yield stress than LP0. The LP90 structure also absorbs a higher level of energy 
calculated out to $20 \%$ strain as listed in Table $1\left(57 \mathrm{MJ} / \mathrm{mm}^{3}\right.$ as compared to $\left.38 \mathrm{MJ} / \mathrm{mm}^{2}\right)$. SEM micrographs of the LP structures after $\sim 10-12 \%$ and of the HP structures after $\sim 12 \%$ strain are shown in Fig. 4A-D.

The HP0 and HP90 structures have different stress-strain curve shapes, though both structures show a load drop after reaching a peak load. This is due to the very different deformation behavior of the two HP geometries dependent on the orientation of the microchannels with respect to the applied load. For the HP0 structure, the interconnection points between orthogonal layers of columns, one of which is circled in Fig. 4C, act as hinges about which the structure shears. The HP90 structure, however, deforms through bending of the high aspect ratio columns - the microchannel walls aligned with the loading direction. These columns are laterally supported by the microchannel walls perpendicular to the loading direction.

\subsubsection{Cyclic Load-Unload-Recovery of Slip Cast Structures}

While large strain monotonic compression is useful in understanding the deformation mechanisms of the structures, in order to better understand the role of the SME in these structures, load-unload-recovery cycles were performed. The cyclic load-unload-recovery curves for $\mathrm{M}$ are plotted in Fig. 3B, along with the monotonic loading curve plotted in a thicker line. The cyclic curves have been plotted such that the accumulation of residual plastic strain is directly visible on the $\mathrm{X}$-axis, with each successive load-unload-recovery cycle beginning at the amount of accumulated residual plastic strain of all previous cycles. SMA detwinning initiates at low loads, prior to the plateau stress at $80 \mathrm{MPa}$. Even in the first cycle to $0.4 \%$ strain $(50 \mathrm{MPa})$, there is $0.2 \%$ residual strain upon unloading, though it is fully recovered when heated through the phase transformation. At higher strains, SMA "plateau" (region with lower strain hardening) is apparent in each curve, becoming progressively steeper, though it initiates at the same plateau 
stress. The load-unload-recovery cycles fit well within the envelope of the monotonic stress strain curve. This shows that the plastic deformation is hardening the structure, and also that the local stress field is very heterogeneous. In earlier cycles only a small volume fraction of the material reaches high enough stress concentrations to plastically deform. However these volume fractions are strengthened by the plasticity for later cycles and increase the hardening rate after the SMA plateau. The volume fractions which have lower stress concentrations have not yet plastically deformed in the later cycles, and contribute to the deformation within the SMA plateau.

In order to investigate these heterogeneities, the maximum strain in each cycle was broken into the following incremental strain components as in $[13,39]$ : the elastic recovery strain $\left(\Delta \varepsilon_{\mathrm{el}}\right)$, the super-elastic recovery strain $\left(\Delta \varepsilon_{\mathrm{se}}\right)$, the thermal recovery strain $\left(\Delta \varepsilon_{\mathrm{th}}\right)$ and the residual plastic strain $\left(\Delta \varepsilon_{\mathrm{pl}}\right)$ which are schematically illustrated in Fig. 3C inset. The elastic recovery strain is calculated by extending the initial linear unloading stiffness to intercept the X-axis. Subtracting the strain at this intercept from the maximum cycle strain is the $\Delta \varepsilon_{\mathrm{el}}$. The $\Delta \varepsilon_{\mathrm{se}}$ is the remaining portion of the unloading strain recovery. The thermal recovery strain $\Delta \varepsilon_{\text {th }}$ is the recovery during heating due to the SME, and the residual strain after heating is the residual plastic strain $\Delta \varepsilon_{\mathrm{pl}}$. The accumulated residual plastic strain $\Delta \varepsilon_{\text {res }}$ is the summation of $\Delta \varepsilon_{\mathrm{pl}}$ for previous cycles. The maximum applied strain $\left(\varepsilon_{\max }\right)$ is designated as the maximum strain in each cycle $(\Delta \varepsilon)$ added to the accumulated residual plastic strain. Individual strain components for $\mathrm{M}$, as well as the accumulated residual plastic strain, are plotted against the maximum applied strain in Fig. 3C.

Other than at very low strains, the thermal recovery strain is the dominant strain component. The thermal recovery strain increases gradually with higher cycle strain $\Delta \varepsilon$ until it reaches a plateau at $\Delta \varepsilon_{\mathrm{th}}=3 \%$ strain. The plastic strain component is small until $4 \%$ strain, where hardening is 
apparent on the monotonic stress-strain curve indicated by the black arrows in Fig. 3B and C. After this point the in-cycle residual plastic strain is $\Delta \varepsilon_{\mathrm{pl}}=\sim 0.7 \%$ strain, and the accumulated residual plastic strain grows significantly, reaching $\Delta \varepsilon_{\text {res }}=7.3 \%$ strain by the end of cycling. In [13], the same prealloyed NiTi powders were used to create structures with $\sim 24-34 \%$ porosity by hot isostatic pressing with spaceholders, and the strain components were also examined. These structures exhibited comparable maximum SMA recovery $\left(\Delta \varepsilon_{\text {th }}=\sim 3.5 \%\right.$ strain $)$, but the residual plastic strain was approximately half of the residual plastic strain in these slip cast structures. The bulk material was also studied in [40], hot isostatic pressed NiTi without spaceholders, had SMA recovery of $4.2 \%$ strain in compression. This study found that the SMA recovery strain decreased with increased plasticity, as is also found here.

Individual incremental strain components for the load-unload-recovery cycles were also examined for LP and HP structures in Fig. 5. Conversely with the stress-strain curves, there is low anisotropy between 0 and 90 structures of the same porosity in the individual strain components. However, the porosity does influence the distribution of the individual strain components. For the LP structures, which have stress-strain curves similar to M, the thermal recovery strain $\Delta \varepsilon_{\text {th }}$ also saturates at $\Delta \varepsilon_{\text {th }}=3 \%$ strain. While the deformation mode is the same, uniaxial compression, the LP structures have higher stress concentrations than in $M$ due to the reduced cross-sectional area due to porosity. The residual plastic strain component is correspondingly higher as compared to $\mathrm{M}$.

However the HP structures exhibit a higher level of thermal recovery than either M or LP structures, increasing to $\Delta \varepsilon_{\mathrm{th}}=\sim 3.5 \%$ strain without plateauing by the end of testing. The elastic recovery and superelastic recovery strain components also increase significantly with maximum applied strain. The bending and shearing modes of the HP structures add geometric elastic 
effects to the linear elastic material behavior of the structure. These large amounts of recoverable deformation perpendicular to the loading axis due to bending and shearing increase the linear recovery of each the elastic, superelastic, and thermal recovery strains. This deformation behavior also accommodates the stress concentrations due to the reduced crosssection area from porosity, and so the plastic strain is not increased as compared to that of M.

\subsection{Modeling of Hierarchical Structures}

\subsubsection{Gibson-Ashby Foam Model}

The Gibson-Ashby model is a set of equations commonly used to calculate a first order approximation of the stiffness and strength of a porous material from the bulk material properties [41]. The model is based on the bending of struts within an isotropic cellular structure. The porous structure stiffness is given as: $E_{G A}=C(1-p)^{2} E_{M}$, where $\mathrm{E}_{\mathrm{M}}$ is the bulk material stiffness, $\mathrm{C}$ is a constant geometric constant for proportionality, and $\mathrm{p}$ is the porosity of the structure. $\mathrm{C}$ is typically taken as unity for equiaxed cell shapes [41]. For open-cell metal structures and polymers, $\mathrm{C}$ is experimentally found to be reduced to 0.5 or even 0.1 [42]. Bansiddhi et al. plotted the stiffness with respect to density of NiTi porous structures from literature from a wide variety of processing methods, including both isotropic and anisotropic porosity. It was found that the $\mathrm{C}=0.3$ was a good fit for the Gibson-Ashby model a majority of the structures [4]. Using $C=0.3$ and the stiffness for hot-isostatically pressed dense NiTi of our prealloyed NiTi powders E $=65 \mathrm{GPa}$ [43], the stiffness calculated from the Gibson-Ashby model is plotted in Fig. 6A as a function of porosity. The stiffness for each of the M, LP0, LP90, HP0, and HP90 structures are also plotted in Fig. 6A. As was found in the previous study [4], regardless of the orientation of porosity (isotropic in $\mathrm{M}$ or anisotropic microchannels in hierarchical structures), the Gibson-Ashby model estimates the stiffness very closely. 


\subsubsection{Finite Element Modeling}

3.4.3.1 Macroscopic FEM of Microchannel Structures. While the elastic stiffness is relatively easy to capture with simple analytical elastic composite models as shown above, the SMA deformation and recovery is not. FEM can be used to capture the influence of the structure geometry on the martensitic variant reorientation, as well as to calculate the stress/strain distribution within the structure. FEM can be difficult to use with SMA foams, as random porosity requires large representative volume units, leading to large numbers of elements and computational time. The structures here, however, are very regular on the macroscale, with predictable channel dimensions and orientations. The geometries of the specimen we examined in compression were limited by experimental constraints. However in FEM we can examine an idealized representative volume element (RVE) for each of the structures (LP0, LP90, HP0, HP90) with periodic boundary conditions, probing the bulk properties of each of these geometries. In addition to examining the loading-unloading-recovery properties on the macroscale, we can also determine the location and magnitude of geometric stress concentrations within each of these structures.

Here we use the Stebner-Brinson SMA model [46] with plasticity [47]. We calibrated the model to the mechanical properties of the monolithic slip cast specimen (Fig. 3A), which is the matrix material in the structures with microchannels. The phase transformation temperatures were taken from DSC values above, and the maximum recoverable martensite, $\gamma$, is $3 \%$ strain. We examine the 4 RVEs illustrated in Fig. 7, which are the RVEs for the LP0, LP90, HP0, and HP90 structures examined experimentally. These RVEs are modeled with a microchannel width of 255 $\mu \mathrm{m}$, and a microchannel wall thickness of $1340 \mu \mathrm{m}$ for LP structure, and $520 \mu \mathrm{m}$ for HP structures (the nominal dimensions of the experimental microchannels and microchannel walls.). 
This results in a microchannel volume fraction of 16 and 33 vol\% for LP and HP samples respectively. These are the ideal microchannel volume fractions, as discussed in section 3.2. For comparison with experimental values, the total RVE structure porosity is used in Fig. 6A, which is the microchannel volume fraction added to the porosity of the matrix, which is $28 \mathrm{vol} \%$ of the matrix. As discussed in section 3.2, this results in a total ideal porosity of $41 \%$ for LP RVEs and 53\% for HP RVEs. A uniform displacement was applied to the face as indicated by the arrows in Fig. 7. The side opposite to the loading face was pinned, while periodic boundary conditions were applied to the faces perpendicular to the applied load. For the periodic boundary conditions, the displacement for each node on a perpendicular face was constrained to the displacement of the corresponding node on the opposite face in both directions perpendicular to the loading direction. Each of the RVEs was modeled by displacing to 5\% strain, unloading, and then heated to recover the SMA deformation.

The macroscopic stress-strain graphs for the structures, as well as for the matrix material, are plotted in Fig. 6B. The stiffnesses and yield stress values from both FEM and experimental measurements are summarized in Table 1, along with the stiffnesses as calculated with the Gibson-Ashby model, while all experimental and modeling stiffness values are plotted in Fig. 6A as a function of porosity. Each of the structures deformed with linear elastic behavior until $0.9 \%$ strain, at which point a SMA plateau is apparent in each curve. The stiffness of the structures decreased with increasing porosity and with loading orientation. The structures loaded parallel to the stacking direction (LP0 and HP0) exhibited stiffnesses of 8.1 and 6.3 GPa respectively, while the structures loaded parallel to the stacking direction (LP90, and HP90) had stiffnesses of 4.1 and 3.1 GPa respectively. The stress onset of the SMA plateau also decreased with the same trend $70 \mathrm{MPa}$ (LP0), $55 \mathrm{MPa}$ (HP0), $35 \mathrm{MPa}$ (LP90), and $27 \mathrm{MPa}$ (HP90). This ordering would 
be expected based on the geometries of the RVEs. The orientation of the microchannels results in two types of compression - compression of columns (LP0 and HP0), and compression of a horizontal "crack" (LP90 and HP90). It is reasonable that the compression of the columns results in higher stiffnesses and higher stress onsets of the SMA reorientation than compression of cracks. Higher stiffness and SMA reorientation onset stress can also be expected for lower porosity samples, as observed with these RVEs. These results also show the limitations of simple homogenized analytical models such as the Gibson-Ashby modeling, which cannot distinguish between the different deformation modes of samples at the same porosity, which is clearly shown in the finite element simulations.

The stiffness and onsets of the SMA plateau agree reasonably well between experimental and modeled values, though with different ordering. The experimental samples have complicated geometries as compared to the RVEs, having both non ideal geometry and porosity. Specifically, the LP90 experimental sample contained a section of matrix without microchannels, decreasing the number of "cracks" in the geometry and strengthening the structure with respect to the ideal RVE as can be clearly seen in Fig. 6A.

With increased loading past $4.5 \%$ strain, the LP0 structure exhibits hardening in the stress-strain curve, indicating initiation of plasticity. In order to prevent geometric instability from occurring in the simulations, all simulations were run to 5\% strain. The LP90 structure also very slightly exhibits hardening right before 5\% strain, and no hardening is visibly apparent in the HP90 curve up to 5\% strain. Upon unloading, the structures recover the linear elastic deformation, and upon heating recover strain from SMA. As expected by the limited hardening apparent in each of the stress strain curves, only a small amount of macroscopic residual strain $(<0.05 \%$ strain $)$ remains after the strain due to the SME is recovered. 
However both the LP90 and HP0 structures exhibit localized residual strains of $1.4 \%$ and $2.4 \%$ strain respectively. This is due to the higher localized strain concentrations of the structures. At 5\% macroscopic strain, the LP90 and HP0 structures exhibited 9.2\% and 9.6\% maximum localized strain respectively. The LP0 and HP90 structures only exhibited 6.0\% and 6.3\% maximum localized strain, resulting in no residual strain after SMA recovery. It is expected that the HP0 would exhibit higher localized stresses as compared to the LP0 structure, as the aspect ratio of the microchannel walls is much higher in the HPO RVE. However the difference between the LP90 and HP90 structure is less intuitive. While the microchannel volume fraction and microchannel wall aspect ratio is also higher for the HP90 RVE as compared to the LP90, this actually results in a relatively sharper crack in the LP90 RVE as compared to HP90, with higher local strain concentrations. The local strain is plotted on each of the RVEs in Fig. 7 in the initial state, at 5\% applied strain, upon unloading, and after SMA recovery through heating.

Unlike the experimental sample, the HP90 structure did not exhibit bending or buckling in the finite element model, highlighting that the experimental sample is not an RVE, but rather a nonsymmetric segment of the material. However the HP0 RVE did exhibited bending. At $4.5 \%$ strain, there is a peak in the stress-strain curve, with a steady drop in load past $4.5 \%$ strain. The structure bends with loading, and after $4.5 \%$ strain initiates buckling. Upon unloading, the HP0 recovers the elastic component of the bending deformation. After heating, the reoriented martensite recovers as well, and the structure straightens to its original shape. The HP0 structure has $0.14 \%$ residual plastic strain after heating, though this is also an insignificant amount of residual plastic strain.

Though maps of the reoriented martensite volume fraction are not displayed here, they track directly with the localized strain maps. At 5\% microscopic strain, regions of the RVE which 
have reached 5\% localized strain have fully reoriented. However regions with lower local strain, $\sim 2.5 \%$ local strain, also have a lower volume fraction of reoriented martensite - around $60 \%$. Regions below $1 \%$ local strain have not reoriented at all. Thus the localized strain can serve as a proxy for depicting the reoriented martensite volume fraction directly. This shows the discrete nature of martensitic reorientation, which initiates due to the local strain rather than the global strain.

\subsubsection{Microscopic FEM of the NiTi-Eutectic Matrix.}

The macroscopic properties of the structures with microchannels can be modeled reasonably well with FEM. The properties of the complex matrix can also be probed by modeling the microstructure. Using an axisymmetric model, a NiTi powder connected with NiTi-Nb eutectic bridges, similar to Fig. 2C, was modeled with the Stebner-Brinson SMA model without plasticity. The NiTi powder phase was modeled as shape memory NiTi with a stiffness of 56 GPa. The material properties and phase transformation temperatures of the NiTi powder were calibrated from compression properties of bulk hot-isostatically pressed NiTi with the same composition as the prealloyed NiTi powders here [38]. The eutectic bridge was modeled as superelastic NiTi with a stiffness of $46 \mathrm{GPa}$. The material properties and phase transformation temperatures of the $\mathrm{NiTi}-\mathrm{Nb}$ eutectic bridge were calibrated from compression properties of a bulk cast NiTi-Nb eutectic [[19]].

The model is shown inset in the top left of Fig. 8, with the material sections delineated. The model is $2 \mathrm{D}$ axisymmetric, with the axisymmetric axis indicated on the left side of the model. For an idealized geometry with perfect symmetry such a model is equivalent to a full $3 \mathrm{D}$ simulation of NiTi powders connected by eutectic bridges. A uniform displacement was applied to the lower bridge side, indicated with a yellow arrow inset in the top left of Fig. 8 . 
Symmetric boundary conditions were applied to the side opposite of loading. The right side opposite of the axisymmetric axis was constrained to remain parallel during loading such that the axisymmetric structure remained cylindrical. The NiTi powder - eutectic bridge composite model was deformed to $2.5 \%$ applied strain, unloaded, and then heated through the SMA transformation. The porosity of the model is $28 \%$, corresponding to the experimental porosity of the slip cast matrix. The area fraction of NiTi powder to eutectic bridge material also agrees with the slip cast matrix, with $16.5 \%$ of the solid model as NiTi-Nb eutectic material. By mass conversion, the $5.3 \mathrm{wt} \%$ of $\mathrm{Nb}$ powders in the experimental powder blend reacted with $12.3 \mathrm{wt} \%$ NiTi powders, resulting in $16.5 \mathrm{vol} \%$ solidified eutectic and $83.5 \mathrm{vol} \%$ solid NiTi powders.

The stress-strain curve for the modeled composite is plotted in Fig. 8. Insets plot the local strain (left) and the volume fraction of reoriented martensite (right) at several points along the stressstrain curve. Reoriented martensite is defined as either transformed martensite from the superelastic eutectic phase, or martensite that has been reoriented from the self-accommodated martensite in the shape memory phase. After initial linear loading with a stiffness of $12 \mathrm{GPa}$, a combined shape memory - superelastic plateau initiates at $0.2 \%$ strain and $25 \mathrm{MPa}$.

Strain is concentrated along the outside of the directly loaded eutectic bridge, radiating through the martensitic NiTi powder. While both phases accommodate strain, the martensitic phase has a lower shape memory yield stress, and contains a higher volume fraction of reoriented martensite. The reorienting NiTi powder shields the core of the loaded eutectic bridge, which remains as austenite at $2.5 \%$ strain. Upon unloading, a majority of the eutectic bridge unloads superelastically. However, the interaction between the two phases creates internal stresses, though the structure is macroscopically unloaded. This prevents the eutectic bridge from fully 
transforming back to austenite. However after heating, the NiTi powder recovers strain, and releases the eutectic phases to fully recover as well.

The modeled stiffness of the shape memory NiTi powder - superelastic eutectic bridge composite is very similar to the experimentally measured $9 \mathrm{GPa}$. The modeled shape memory / superelastic plateau, however, initiates at lower loads than experimentally. In the model, the upper eutectic bridge is a belt when the $2 \mathrm{D}$ model is rotated about the axisymmetric axis, rather than the four bridges that would be present in this type of node model. This increases the crosssectional area of the structure, decreasing the average load. Within the experimental slip cast structure, the eutectic bridges are also varied in shape, size, and connectivity between NiTi powders. Many bridges are shorter and wider, which would decrease the stress concentration and raise the initiation stress of the shape memory plateau.

Though outside the scope of this present study, larger RVEs could be explored to account for the nonuniformity of the powders and bridges. These results provide promise for use of FE as a tool for microstructural material design of these systems. Varying the volume fraction of the eutectic bridge phase can be used as a design tool to optimize the amount of $\mathrm{Nb}$ which should be used during processing, as higher wt\% of $\mathrm{Nb}$ in the $\mathrm{NiTi}-\mathrm{Nb}$ powder blend directly results in a higher vol\% of the eutectic phase. FE can also be used to optimize the NiTi powder size with respect to the eutectic bridge volume fraction for slip cast matrix design.

\section{Conclusions}

SMA NiTi-Nb porous structures were created with hierarchical porosity, consisting of finer, equiaxed and homogenously distributed porosity and larger, orthogonally arranged and interconnected microchannels. Mg ribbons were orthogonally stacked into a scaffold, in the 
empty space of which a NiTi-Nb powder slurry with a PVA binder was slip cast. The dried $\mathrm{NiTi}-\mathrm{Nb} / \mathrm{Mg}$ green body subjected to a four-step vacuum heat treatment, in which the PVA binder was first thermally debound, the magnesium was then evaporated, and finally the NiTi-Nb matrix was solid- and then liquid-phase sintered. A small amount of the liquid NiTi-Nb eutectic phase formed during liquid phase sintering wicked between NiTi powders forming eutectic bridges - without filling the microchannels created by the $\mathrm{Mg}$ evaporation - consisting of a $\mathrm{Nb}$ containing NiTi matrix and Ni-and Ti-containing $\mathrm{Nb}$ lamellae.

In addition to the monolithic slip cast structure, structures with four different geometries of microchannels were subjected to compressive testing: a low $(\sim 12 \%)$ microchannel porosity and a high $(\sim 30 \%)$ microchannel porosity, with the microchannel stacking direction parallel or perpendicular to the loading direction. The monolithic slip cast structure densified uniformly up to $28 \%$ compressive strain without any spalling or cracking apparent in the structure, with a stiffness of $9 \mathrm{GPa}$ and a shape memory yield stress of $80 \mathrm{MPa}$. The structures with microchannels exhibited mechanical anisotropy, with stiffnesses ranging from 4 to $7 \mathrm{GPa}$, which is between that of trabecular and cortical bone, and yield strengths from 20 to $65 \mathrm{MPa}$. All of the structures exhibited excellent SMA properties, recovering at least 3\% strain through SMA recovery even after large amounts of residual plastic strain accumulated during multiple loadunload cycles. The structures compressive strength, stiffnesses and high recoverable strains are appropriate for bone implant applications.

In FEM, the structures with low volume fraction of microchannels structures were found to have lower localized strain concentrations than structures with high volume fraction of microchannels. The structure with a high volume fraction of microchannels aligned with the stacking direction parallel to loading exhibited bending in the microchannel walls, as in experimental compression 
testing. On the microscopic level, modeling a NiTi powder connected by NiTi-Nb bridges well replicated the stiffness, though the onset stress of the SMA plateau was lower in FEM.

Martensitic reorientation was dominated by the martensitic NiTi powder phase, which has a lower shape memory yield plateau stress than the superelastic eutectic phase. In the superelastic eutectic bridge phase the strain was concentrated around the outside of the bridge while the core of the bridge was shielded from transformation by the NiTi powder. Internal stresses were also present between the two phases after unloading, as the eutectic phase was constrained by the martensitic phase, though they were relieved upon heating. Varying both the volume fraction and geometry of the eutectic bridge with respect to the NiTi powder size can be used as a design tool to optimize the NiTi-Nb powder blend ration. With proper calibration of the material properties of both phases, FE can also be used to optimize the NiTi powder composition to fully design the slip cast matrix material and structures.

\section{Acknowledgements}

This research was supported by the Office of Army Research (W911NF-12-1-0013/P00002), as well as the Department of Energy Basic Energy Science (DE-SC0010594). C. Bewerse also gratefully acknowledges Government support under DoD, Air Force Office of Scientific Research, National Defense Science and Engineering Graduate (NDSEG) Fellowship, 32 CFR 168a. Ms. P. Zhu is acknowledged for her assistance with FEM, and Mr. Partha Paul for help with the manuscript.

\section{References}

[1] Y. Bellouard, Shape memory alloys for microsystems: A review from a material research perspective, Mater. Sci. Eng. A. (2008) 481-482, 582-589. 
[2] M.H. Elahinia, M. Hashemi, M. Tabesh, S.B. Bhaduri, Manufacturing and processing of NiTi implants: A review, Prog. Mater. Sci. 57 (2011), 911-946.

[3] T. Tadaki, K. Otsuka, K. Shimizu, Shape Memory Alloys, Annu. Rev. Mater. Sci. 18 (1988), $25-45$.

[4] A. Bansiddhi, T.D. Sargeant, S.I. Stupp, D.C. Dunand, Porous NiTi for bone implants: a review, Acta Biomater. 4 (2008), 773-82.

[5] N. Morgan, Medical shape memory alloy applications - the market and its products, Mater. Sci. Eng. A. 378 (2004), 16-23.

[6] M.H. Wu, L.M. Schetky, Industrial Applications for Shape Memory Alloys, Proceedings of the International Conference on Shape Memory and Superelastic Technologies, Pacific Grove, California, USA, (2000) pp. 171-182.

[7] G. Song, N. Ma, H.N. Li, Applications of shape memory alloys in civil structures, Eng. Struct. 28 (2006), 1266-1274.

[8] V. Karageorgiou, D. Kaplan, D, Porosity of 3D biomaterial scaffolds and osteogenesis, Biomaterials. 26 (2005), 5474-91.

[9] G. Ryan, A. Pandit, D.P. Apatsidis, Fabrication methods of porous metals for use in orthopaedic applications, Biomaterials. 27 (2006), 2651-70.

[10] M.B. Nasab, M. R. Hassan, B. Sahari, Metallic Biomaterials of Knee and Hip - A Review, Trends Biomater. Artif. Organs. 24 (2010), 69-82. 
[11] A. Bansiddhi, D.C. Dunand, Shape-memory NiTi foams produced by replication of $\mathrm{NaCl}$ space-holders, Acta Biomater. 4 (2008), 1996-2007.

[12] A. Bansiddhi, D.C. Dunand, Shape-memory NiTi foams produced by solid-state replication with NaF, Intermetallics. 15 (2007), 1612-1622.

[13] A.J. Neurohr, D.C. Dunand, Shape-memory NiTi with two-dimensional networks of microchannels, Acta Biomater. 7 (2011), 1862-72.

[14] T. Aydoğmuş, Ş. Bor, Processing of porous TiNi alloys using magnesium as space holder, J. Alloys Compd. 478 (2009), 705-710.

[15] T. Aydoğmuş, Ş. Bor, Enhanced Sintering of TiNi Shape Memory Foams under Mg Vapor Atmosphere, Metall. Mater. Trans. A 43 (2012), 5173-5181.

[16] G. İpek Nakaş, A.F. Dericioğlu, Ş. Bor, Monotonic and cyclic compressive behavior of superelastic TiNi foams processed by sintering using magnesium space holder technique, Mater. Sci. Eng. A. 582 (2013), 140-146.

[17] G.O. Cook, C.D. Sorensen, Overview of transient liquid phase and partial transient liquid phase bonding, J. Mater. Sci. 46 (2011), 5305-5323.

[18] C. Bewerse, L.C. Brinson, D.C. Dunand, Microstructure and Mechanical Properties of asCast Quasibinary NiTi-Nb Eutectic Alloy, Mater. Sci. Eng. A. 627 (2014), 360-368. 
[19] C. Bewerse, L.C. Brinson, D.C. Dunand, NiTi with 3D-interconnected microchannels produced by liquid phase sintering and electrochemical dissolution of steel tubes. J. Mater. Process. Technol. 214 (2014), 1895-1899.

[20] M. Piao, S. Miyazaki, K. Otsuka, N. Nishida, N, Effects of Nb Addition on the Microsctruture of Ti-Ni Alloys, Mater. Trans. JIM 33 (1992), 337-345.

[21] A. Bansiddhi, D.C. Dunand, Shape-memory NiTi - Nb foams, J. Mater. Res. 24 (2009), $2107-2117$.

[22] A. Bansiddhi, D.C. Dunand, Niobium Wires as Space Holder and Sintering Aid for Porous NiTi, Adv. Eng. Mater. 13 (2011), 301-305.

[23] D.S. Grummon, J. Shaw, J. Foltz, Fabrication of cellular shape memory alloy materials by reactive eutectic brazing using niobium, Mater. Sci. Eng. A 438-440 (2006), 1113-1118.

[24] D.S. Grummon, K.B. Low, J. Foltz, J.A. Shaw, A New Method for Brazing Nitinol Based on the Quasibinary TiNi-Nb System, American Institute of Aeronautics and Astronautics (2007), pp. $1-7$.

[25] K.B. Low, D.S. Grummon, J. Foltz, J. Shaw, Eutectic Liquid Formation in the NiTi-Nb System: a New Joining Method for Nitinol. Proc. Int. Conf. Shape Mem. Superelastic Technol (2008), 3323-3330.

[26] X.G. Song, J. Cao, Y.Z. Liu, J.C. Feng, Brazing high Nb containing TiAl alloy using TiNi$\mathrm{Nb}$ eutectic braze alloy, Intermetallics 22 (2012), 136-141. 
[27] Liu, Y.Z., Zhang, L.X., Liu, C.B., Yang, Z.W., Li, H.W., Feng, J.C., 2011. Brazing C/SiC composites and $\mathrm{Nb}$ with TiNiNb active filler metal. Sci. Technol. Weld. Join. 16, 193-198.

[28] D.W. Richerson, Modern Ceramic Engineering, Marcel Dekker, New York, NY, 1982.

[29] S. Ohkawa, K. Ishii, M. Uo, T.Sugawara, F. Watari, Slip Casting of Titanium Powder for Dental Prosthetic Appliances, Mater. Trans. 45 (2004), 1132-1139.

[30] A. Battikhi, Drain Casting and Combustion Synthesis of Textured Ni-Ti Interfaces, San Diego State University (2013).

[31] Q. Xu, B. Gabbitas, S. Matthews, D. Zhang, The development of porous titanium products using slip casting, J. Mater. Process. Tech. 213 (2013), 1440-1446.

[32] J. Li, H. Yang, H. Wang, J. Ruan, Low elastic modulus titanium-nickel scaffolds for bone implants, Mater. Sci. Eng. C 34 (2014), 110-114.

[33] H. Matsuno, A. Yokoyama, F. Watari, M. Uo, T. Kawasaki, Biocompatibility and osteogenesis of refractory metal implants, titanium, hafnium, niobium, tantalum and rhenium, Biomaterials 22 (2001), 1253-62.

[34] K.W. Ng, H.C. Man, T.M. Yue, Characterization and corrosion study of NiTi laser surface alloyed with Nb or Co, Appl. Surf. Sci. 257 (2010), 3269-3274.

[35] S.R. Kalidindi, A. Abusafieh, E. El-Danaf, Accurate characterization of machine compliance for simple compression testing, Exp. Mech. 37 (1997), 210-215. 
[36] C.M. Jackson, H.J. Wagner, R.J. Wasilewski, 55-Nitinol-The Alloy with a Memory: Its Physical Metallurgy, Properties, and Applications. Washington, DC (1972).

[37] K.S. Katti, Biomaterials in total joint replacement, Colloids Surf. B. Biointerfaces. 39 (2004), 133-42.

[38] R. Vaidyanathan, M.A.M. Bourke, D.C. Dunand, Phase fraction, texture and strain evolution in superelastic NiTi and NiTi-TiC composites investigated by neutron diffraction, Acta Mater. 47 (1999), 3353-3366.

[39] A.J. Neurohr, D.C. Dunand, Shape-memory NiTi with two-dimensional networks of microchannels, Acta Biomater. 7(2011), 1862-72.

[40] K. Fukami-Ushiro, D.C. Dunand, NiTi and NiTi-TiC Composites : Part III. Shape-Memory Recovery, Metall. Mater. Trans. A 27A (1996), 193-203.

[41] L.J. Gibson, M.F. Ashby, Cellular Solids: Structure and Properties, 2nd ed. Cambridge University Press, Cambridge, United Kingdom, 1997.

[42] H. Hagiwara, D.J. Green, Elastic Behavior of Open-Cell Alumina, J. Am. Ceram. Soc. 70 (1987), 811-815.

[43] K. Fukami-Ushiro, D. Mari, D.C. Dunand, NiTi and NiTi-TiC Composites: Part II. Compressive Mechanical Properties, Metall. Mater. Trans. A 27A(1996), 183. 


\section{List of Fig. Captions}

Fig. 1 - Photographs of A) The plaster mold for slip casting, with grooves for alignment of $\mathrm{Mg}$ ribbons and B) the HP0 green sample. Schematic illustrations of the hierarchical slip cast structures: C) LP0, D) LP90, E) HP0, and F) HP90, as well as G) a schematic of the full HP sample with the HP0 and HP90 structures indicated.

Fig. 2: SEM micrographs of the HP90 structure. A) The microchannel surface is exposed by EDM sectioning. Six orthogonal microchannels connect to the exposed perpendicular microchannel through windows (four of which are marked with arrows). A closed interconnection window is circled. Dashed lines indicate the interface between the machined surfaces and the microchannel surface. B) The as-cast slip cast surface showing irregular pores (marked with arrows) and a larger void (outlined with dotted line). C) eutectic bridge between NiTi powders outlined with a dashed line; $\mathrm{Nb}$ particle on NiTi powder surface is marked with arrow, and D) magnified from (C), showing $\mathrm{Nb}$ lamellae (outlined with solid line).

Fig. 3: A) The monotonic stress-strain curves plotted for each M, LP90, LP0, HP90, and HP0 structures. The full HP90 stress-strain curve out to 55\% strain is inset. B) The M load-unloadrecovery cycle stress-strain curves (with the monotonic stress-strain curve superimposed in bold blue color) and $\mathrm{C}$ ) the incremental elastic recovery ( $\Delta \varepsilon \mathrm{el})$, superelastic recovery $(\Delta \varepsilon s \mathrm{e})$, thermal recovery $(\Delta \varepsilon$ th) and plastic strain $(\Delta \varepsilon \mathrm{pl})$ as a function of the maximum applied strain for $\mathrm{M}$, with a schematic inset of the strain components on a stress-strain curve. The red arrow in the schematic represents strain recovery though heating. The black arrows in B) and C) indicate the strain at which secondary hardening initiates. 
Fig. 4: SEM micrographs of the A) LP0 structure after 9.5\% residual plastic strain, B) the LP90 structure after $9 \%$ residual plastic strain, C) the HPO structure after $9 \%$ residual plastic strain, and D) the HP90 structure after $7.5 \%$ residual plastic strain.

Fig. 5: Incremental elastic recovery $(\Delta \varepsilon \mathrm{el})$, superelastic recovery $(\Delta \varepsilon s e)$, thermal recovery $(\Delta \varepsilon$ th $)$ and plastic strain $(\Delta \varepsilon p l)$ as a function of the maximum applied strain for A) LP and B) HP structures.

Fig. 6: A) The stiffness for M, LP0, LP90, HP90, and HP0 are plotted along with the estimated stiffness from Gibson-Ashby model. Experimental stiffnesses are plotted with filled circles, while FEM stiffnesses are plotted with empty circles. B) Stress-strain curves for each of the RVE's, as well as for the matrix material. The strain recovery due to heating is slightly offset from $0 \mathrm{MPa}$ to display all of the structures.

Fig. 7: Local strain plotted on the RVEs for each LP0, LP90, HP0, and HP90 structures in the initial state, after loading to 5\% applied strain, upon unloading, and after shape memory recovery through heating. The applied displacement is indicated by yellow arrows in the initial state.

Fig. 8: The stress-strain curve for the axisymmetric model of a NiTi powder connected by eutectic bridges plotted with the experimental slip cast matrix M. Insets plot the local strain (left) and the volume fraction of reoriented martensite (right) at several global strain levels. After unloading, strain is fully recovered upon heating. Inset in the top left is the microscopic FEM of a NiTi powder connected with eutectic bridges. The NiTi powder is modeled with shape memory NiTi material properties, while the bridges are modeled with superelastic NiTi material properties. 

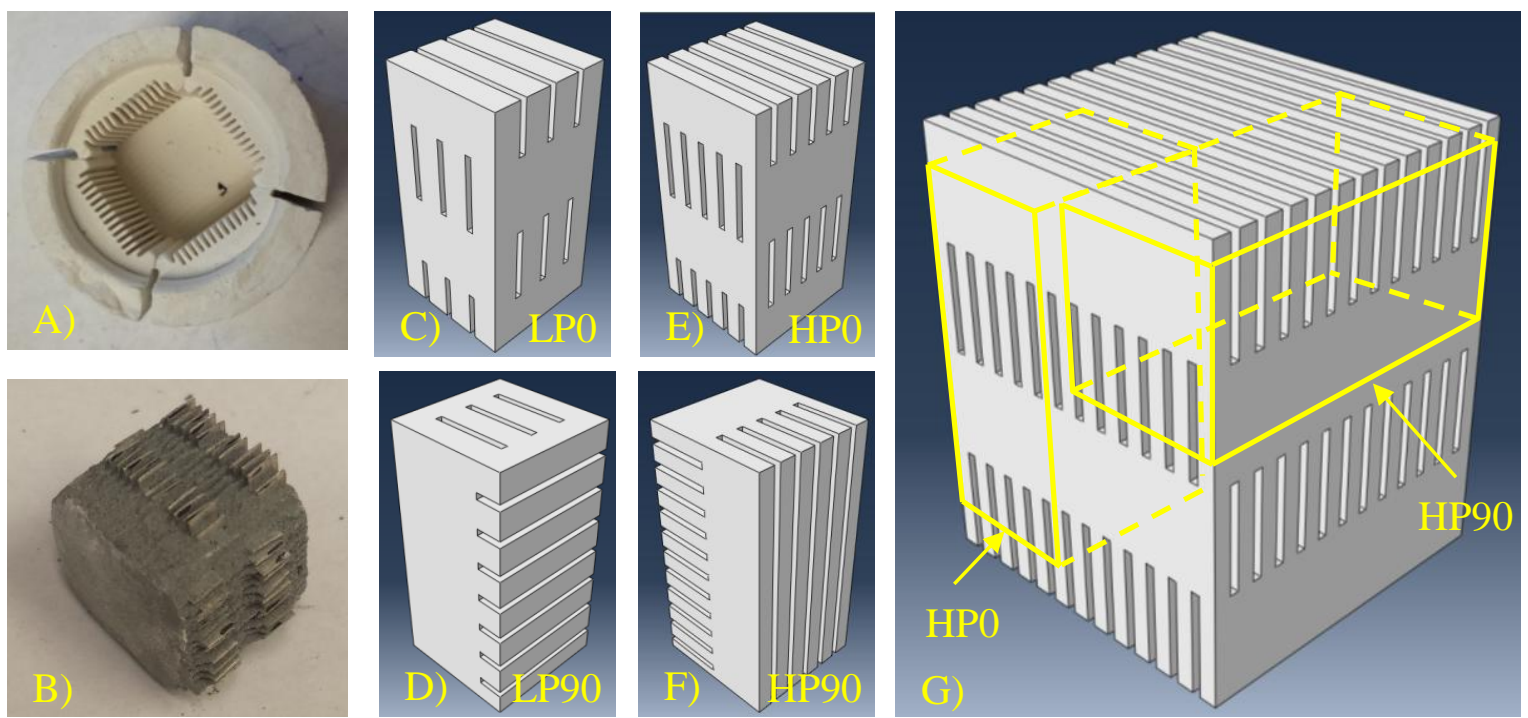

Figure 1 Photographs of A) The plaster mold for slip casting, with grooves for alignment of $\mathrm{Mg}$ ribbons and $\mathrm{B}$ ) the HP0 green sample, and Schematic illustrations of the hierarchical slip cast structures: C) LP0, D) LP90, E) HP0, and F) HP90, as well as G) a schematic of the full HP sample with the HP0 and HP90 structures indicated. 

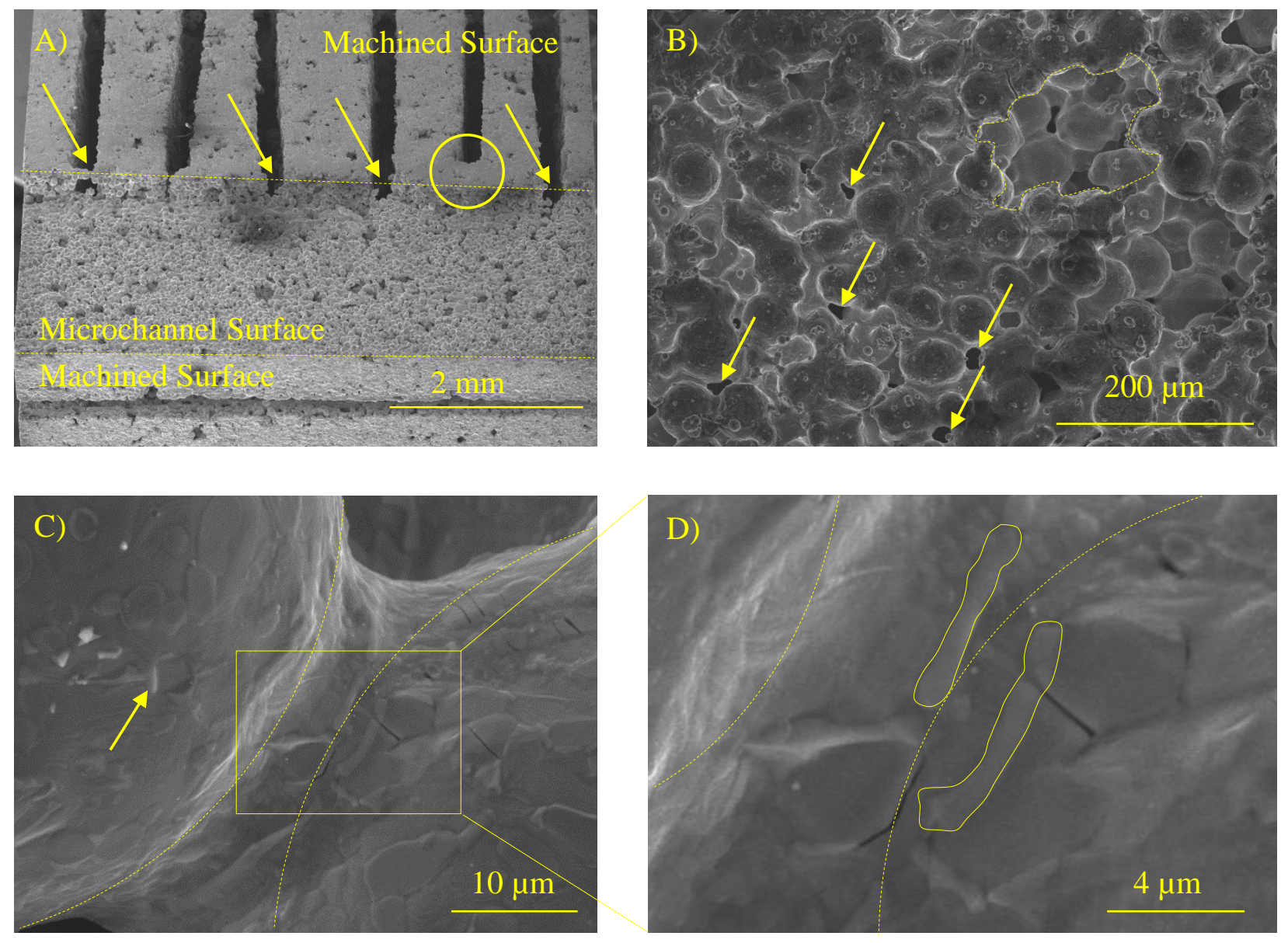

Figure 2: SEM micrographs of the HP90 structure. A) The microchannel surface is exposed by EDM sectioning. Six orthogonal microchannels connect to the exposed perpendicular microchannel through windows (four of which are marked with arrows). A closed interconnection window is circled. Dashed lines indicate the interface between the machined surfaces and the microchannel surface. B) The as-cast slip cast surface, C) eutectic bridge between NiTi powders outlined with a dashed line, and D) magnified from (C), showing the $\mathrm{Nb}$ lamellae (outlined with solid line) 

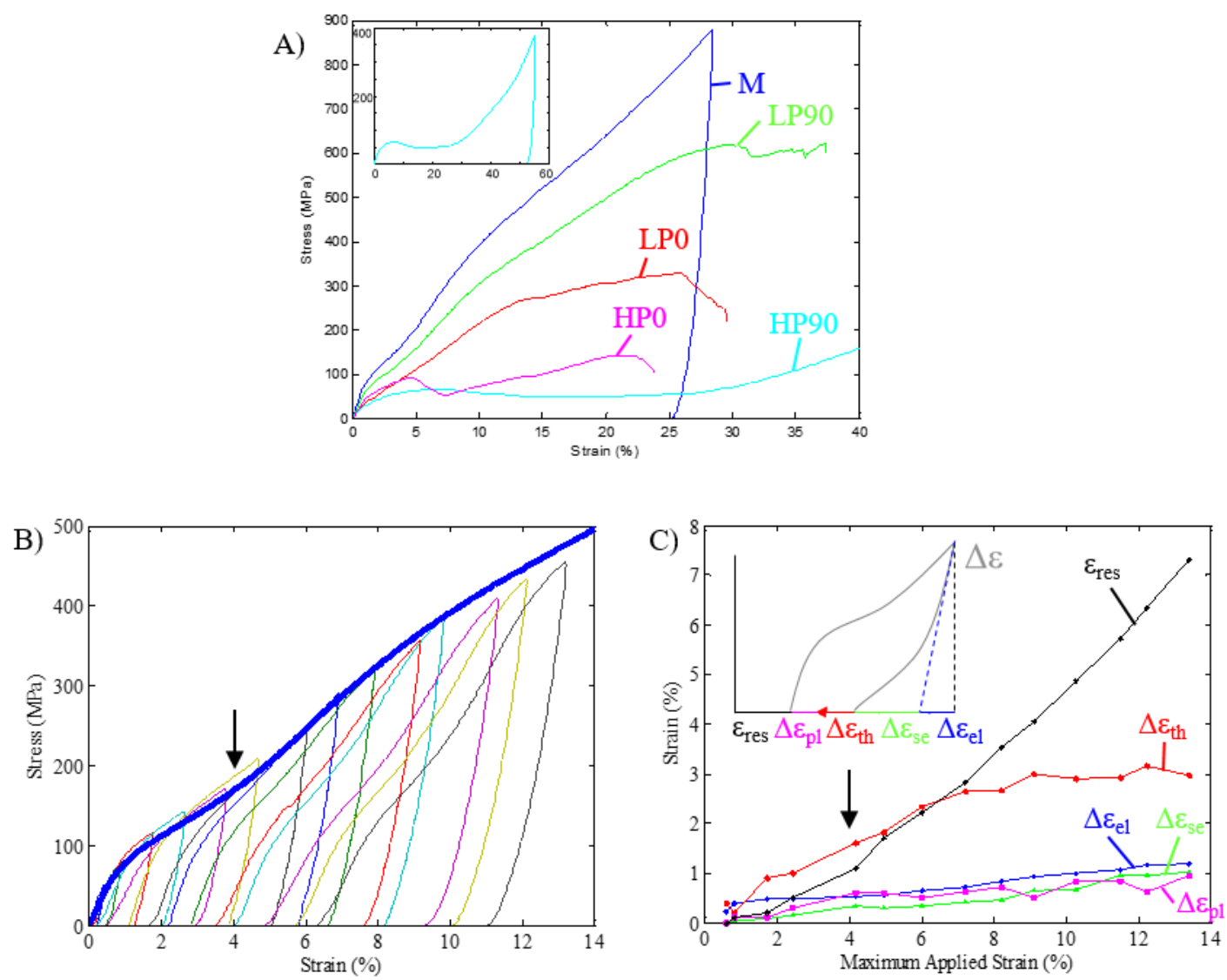

Figure 3: A) The monotonic stress-strain curves plotted for each M, LP90, LP0, HP90, and HP0 structures. The full HP90 stress-strain curve out to $55 \%$ strain is inset. B) The M load-unload-recovery cycle stress-strain curves and $\mathrm{C})$ the incremental elastic recovery $\left(\Delta \varepsilon_{\mathrm{el}}\right)$, superelastic recovery $\left(\Delta \varepsilon_{\mathrm{se}}\right)$, thermal recovery $\left(\Delta \varepsilon_{\mathrm{th}}\right)$ and plastic strain $\left(\Delta \varepsilon_{\mathrm{pl}}\right)$ as a function of the maximum applied strain for $\mathrm{M}$, with a schematic inset of the strain components on a stress-strain curve. The red arrow in the schematic represents strain recovery though heating. The black arrows in B) and C) indicate the strain at which secondary hardening initiates 

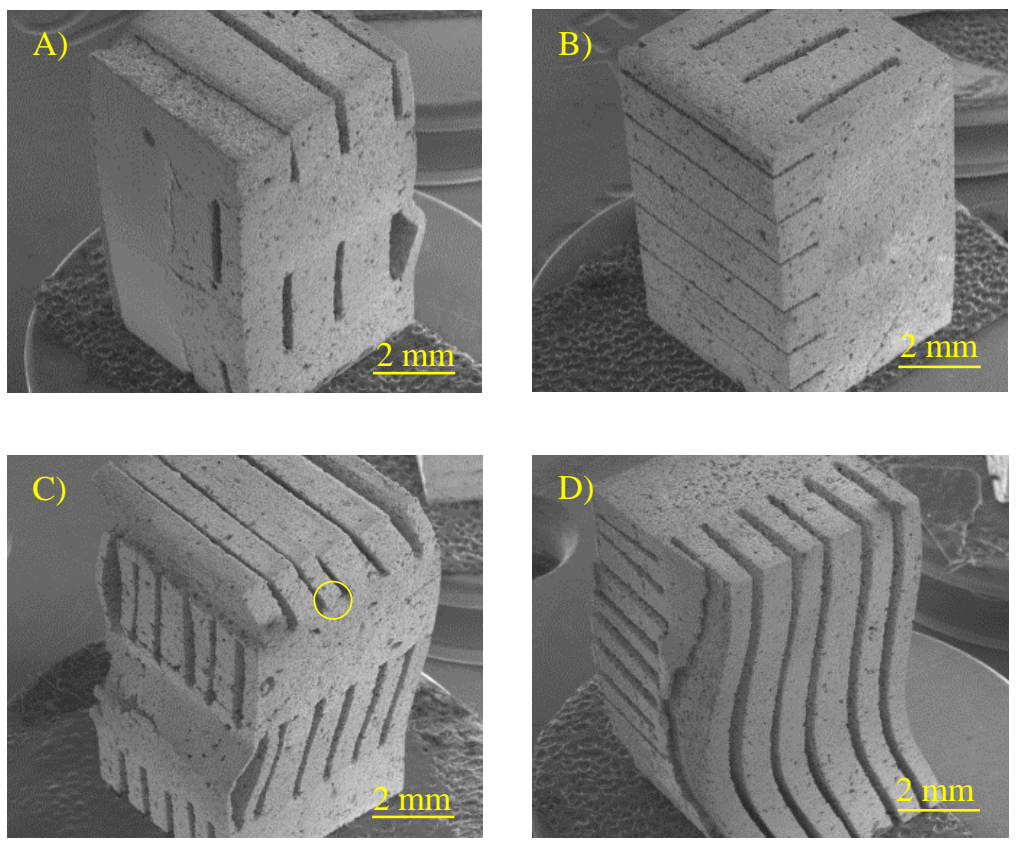

Figure 4: SEM micrographs of the A) LP0 structure after 9.5\% residual plastic strain, B) the LP90 structure after 9\% residual plastic strain, C) the HP0 structure after 9\% residual plastic strain, and D) the HP90 structure after $7.5 \%$ residual plastic strain. 

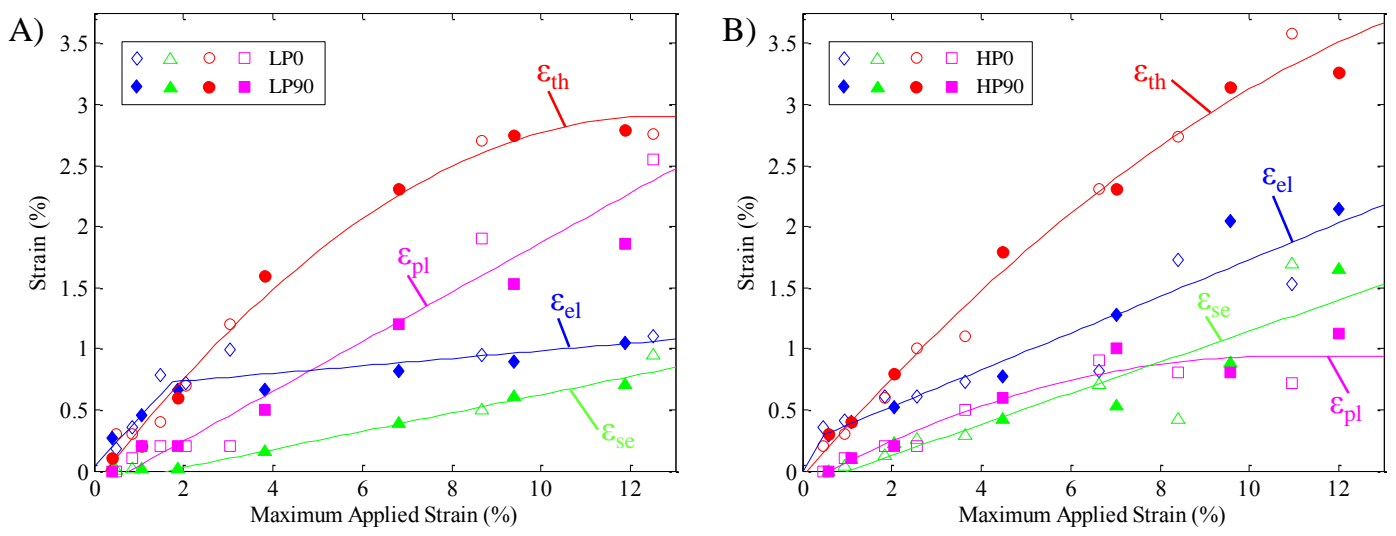

Figure 5: Incremental elastic recovery $\left(\Delta \varepsilon_{\mathrm{el}}\right)$, superelastic recovery $\left(\Delta \varepsilon_{\mathrm{se}}\right)$, thermal recovery $\left(\Delta \varepsilon_{\mathrm{th}}\right)$ and plastic strain $\left(\Delta \varepsilon_{\mathrm{pl}}\right)$ as a function of the maximum applied strain for A) LP and B) HP structures 

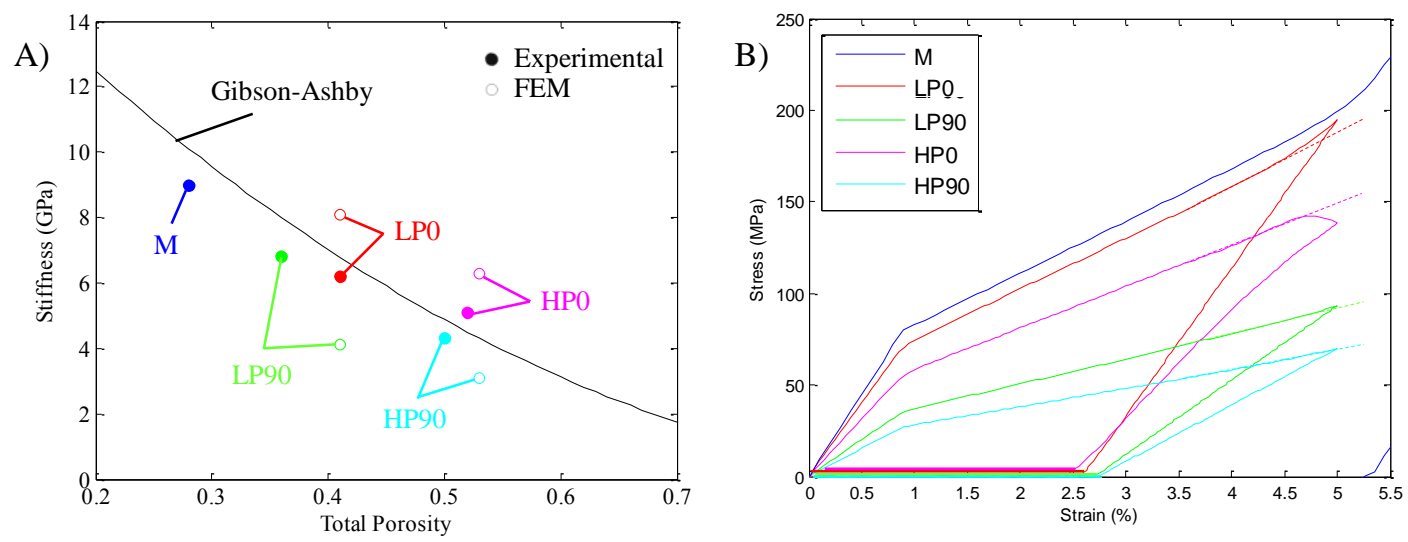

Figure 6: A) The stiffness for M, LP0, LP90, HP90, and HP0 are plotted along with the estimated stiffness from Gibson-Ashby model. Experimental stiffnesses are plotted with filled circles, while FEM stiffnesses are plotted with empty circles. B) Stress-strain curves for each of the RVE's, as well as for the matrix material. The strain recovery due to heating is slightly offset from $0 \mathrm{MPa}$ to display all of the structures. 


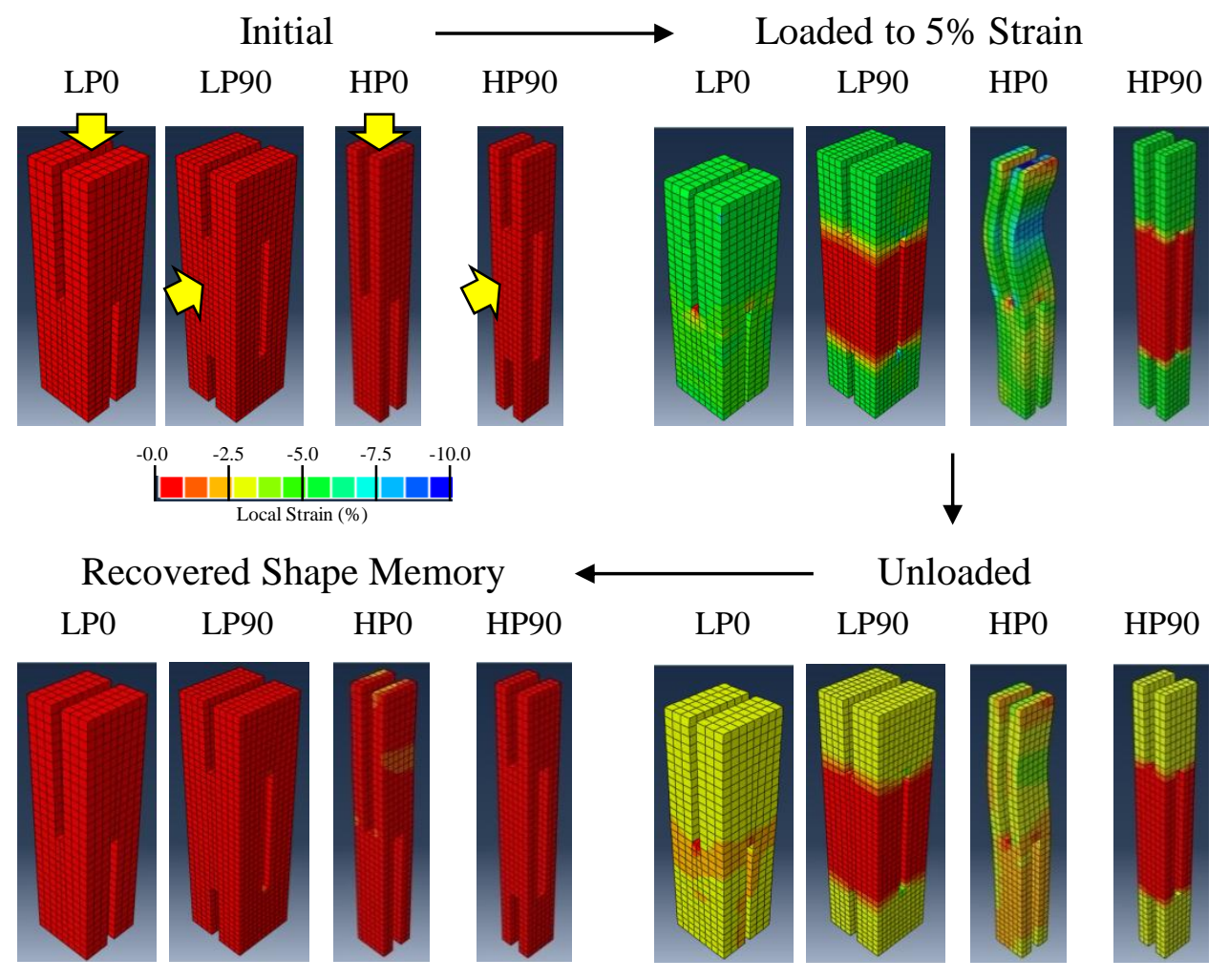

Figure 7: Local strain plotted on the RVEs for each LP0, LP90, HP0, and HP90 structures in the initial state, after loading to 5\% applied strain, upon unloading, and after shape memory recovery through heating. The applied displacement is indicated by yellow arrows in the initial state 


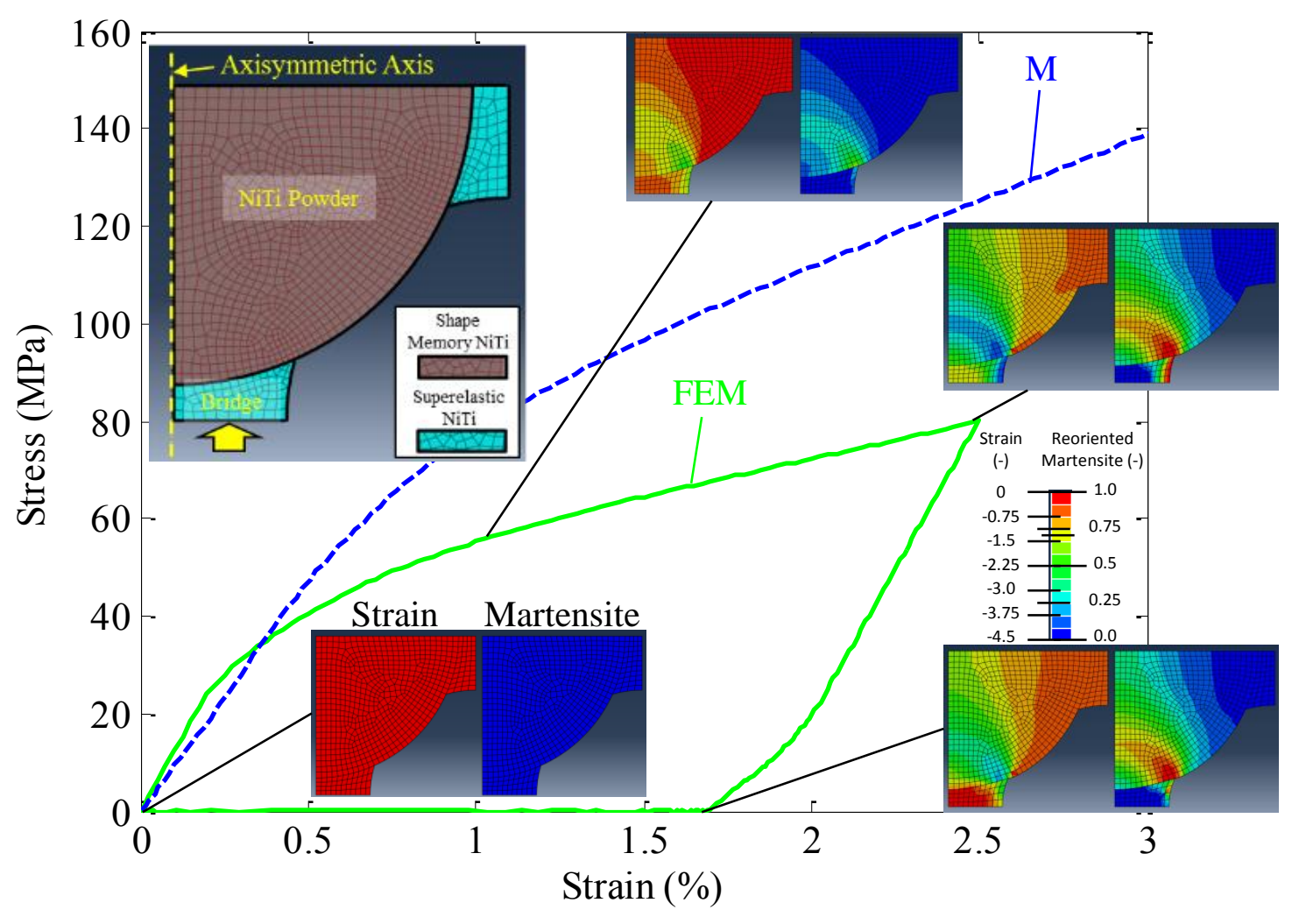

Figure 8: The stress-strain curve for the axisymmetric model of a NiTi powder connected by eutectic bridges plotted with the experimental slip cast matrix M. Insets plot the local strain (left) and the volume fraction of reoriented martensite (right) at several global strain levels. After unloading, strain is fully recovered upon heating. Inset is the top left is the microscopic FEM of a NiTi powder connected with eutectic bridges. The NiTi powder is modeled with shape memory NiTi material properties, while the bridges are modeled with superelastic NiTi material properties 


\begin{tabular}{|c|ccc|c|c|c|c|c|c|}
\hline \multirow{2}{*}{ Structure } & \multicolumn{3}{|c|}{$\begin{array}{c}\text { Porosity } \\
(\%)\end{array}$} & \multicolumn{3}{c|}{$\begin{array}{c}\text { Stiffness } \\
(\mathrm{GPa})\end{array}$} & \multicolumn{2}{c|}{$\begin{array}{c}\text { Yield } \\
\text { Strength } \\
(\mathrm{MPa})\end{array}$} & $\begin{array}{c}\text { Energy } \\
\text { Absorbed } \\
\left(\mathrm{MJ} / \mathrm{mm}^{3}\right)\end{array}$ \\
\cline { 2 - 8 } & Matrix & Channel & Total & Expt & GA & FEM & Expt & FEM & \\
\hline M & 28 & 0 & 28 & 9.0 & 10.1 & - & 80 & - & 73 \\
LP0 & 29 & 12 & 41 & 6.2 & 6.7 & 8.1 & 35 & 70 & 38 \\
LP90 & 24 & 12 & 36 & 6.8 & 8.0 & 4.1 & 65 & 35 & 57 \\
HP0 & 19 & 33 & 52 & 5.1 & 4.5 & 6.3 & 51 & 55 & 10 \\
HP90 & 23 & 27 & 50 & 4.3 & 4.9 & 3.1 & 20 & 27 & 17 \\
\hline
\end{tabular}

Table 1: Physical and Mechanical Properties of Slip Cast Structures 
Loaded to $5 \%$ Strain

LP0

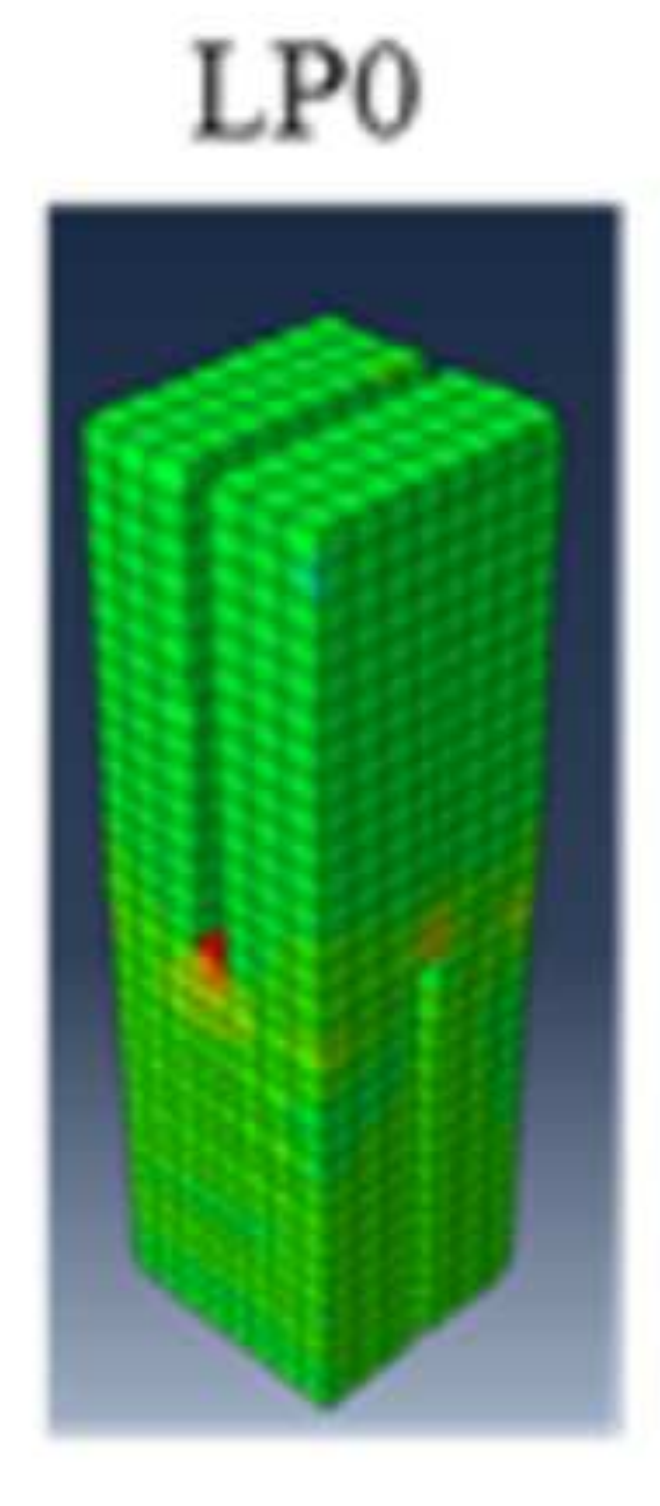

LP90

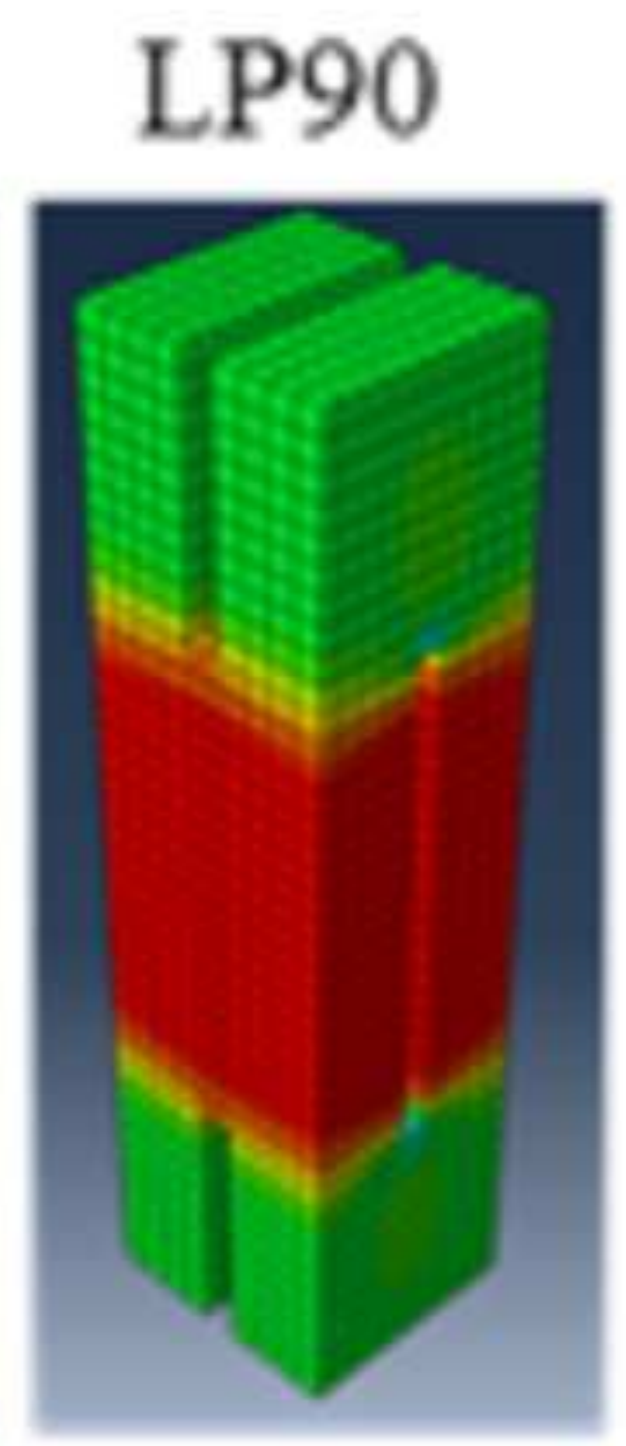

HP0

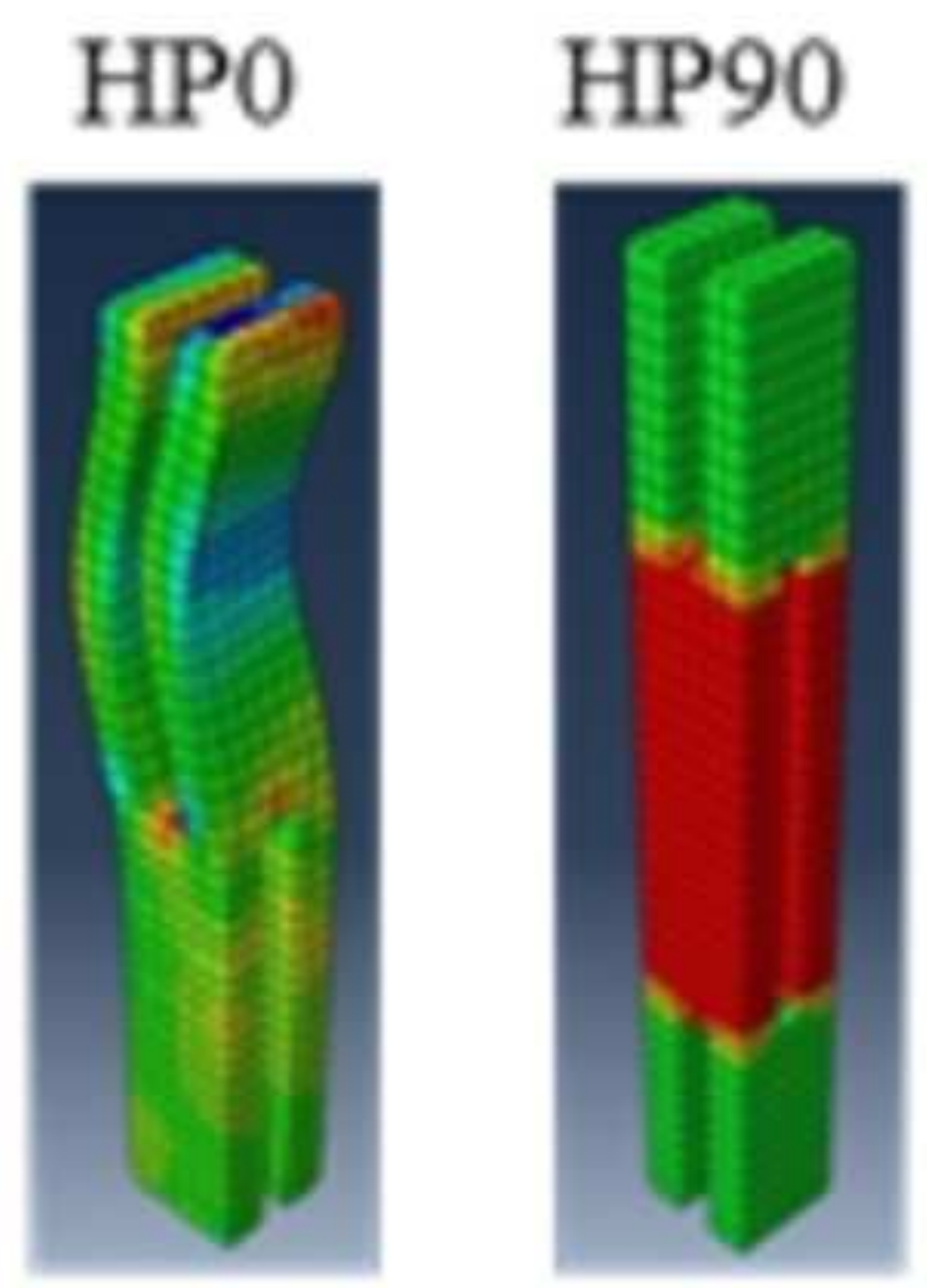
HP90
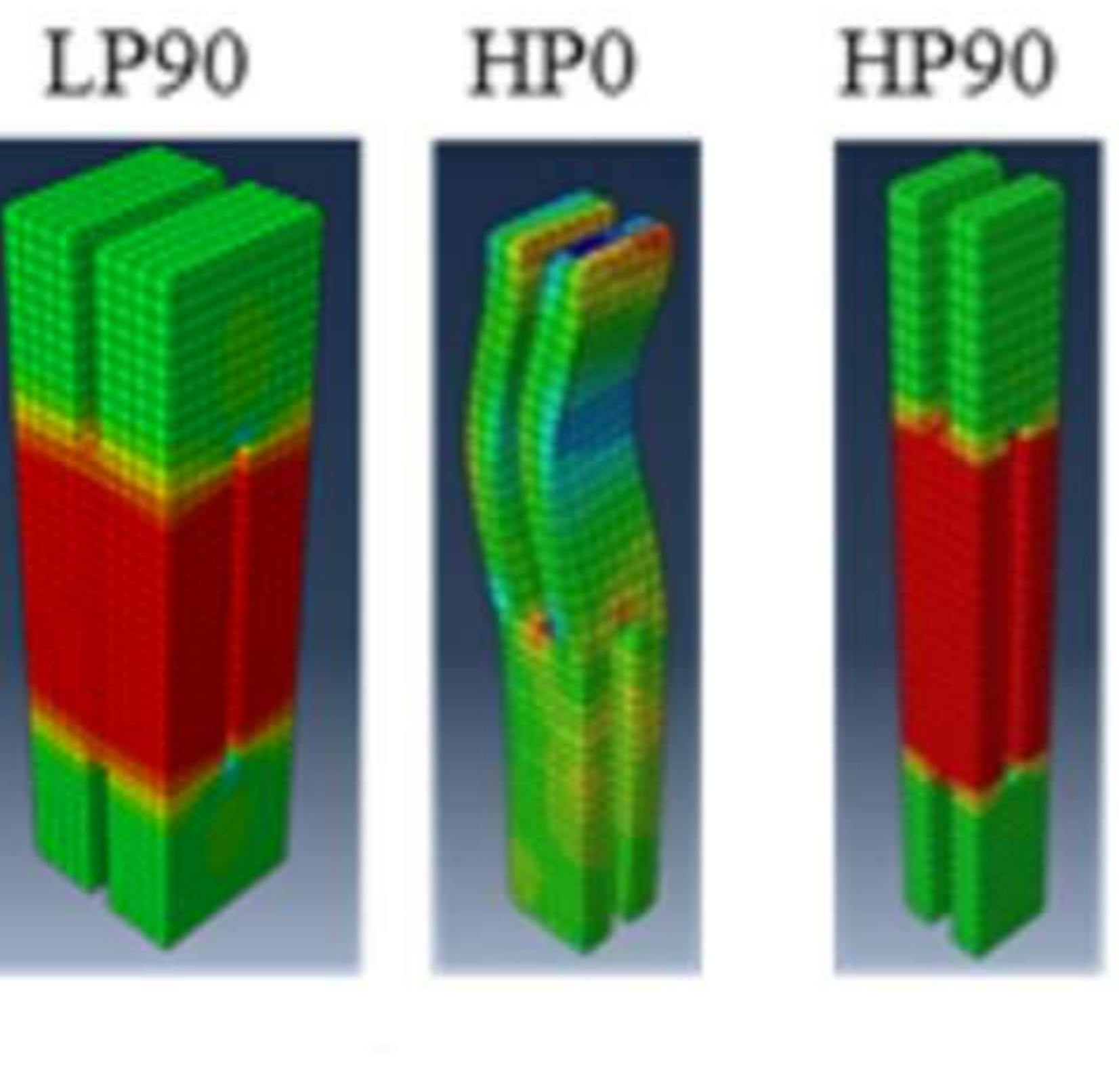

$$
\text { . }
$$

